\title{
Bcl-xl and Mcl-1 are the major determinants of the apoptotic response to dual PI3K and MEK blockage
}

\author{
ELINA JOKINEN and JUSSI P. KOIVUNEN

\begin{abstract}
Department of Medical Oncology and Radiotherapy and Medical Research Center Oulu, Oulu University Hospital and University of Oulu, Oulu, PB22 90029 OYS, Finland
\end{abstract}

Received February 25, 2015; Accepted April 6, 2015

DOI: 10.3892/ijo.2015.3071

\begin{abstract}
The dual targeting of PI3K-AKT-mTOR and Ras-Raf-MEK-ERK pathways is a potential anticancer therapy, but unfortunately, the response rate has been low in early phase clinical trials. Pre-clinical models have suggested that an apoptotic response to dual PI3K and MEK targeting is relatively rare and understanding apoptotic avoidance could lead to increased clinical efficiency. This study investigated solid cancer cell lines, which are known to be sensitive to dual PI3K and MEK inhibition but to have a limited apoptotic response. The cells were exposed to dual PI3K and MEK blockage in combination with a panel of additional pharmacological agents and cytotoxicity and apoptosis were analyzed. Our results indicated that the BH3 mimetic ABT-263, the HDAC inhibitor entinostat and the multikinase inhibitor dasatinib increased the cytotoxicity and apoptotic response of dual PI3K and MEK targeting. Furthermore, ABT-263 and entinostat was able to induce apoptosis in combination with single agent PI3K and MEK inhibitors. Protein expression, immunoprecipitation and siRNA knockdown models suggested that Bcl-xl and Mcl-1 were the most important factors circumventing PI3K and/or MEK inhibition-mediated apoptosis. The results suggest that the cytotoxicity of PI3K and/or MEK inhibitor treatments can be augmented by combinatory approaches targeting antiapoptotic mediators Bcl-xl and Mcl-1.
\end{abstract}

\section{Introduction}

In solid malignancies, the PI3K-AKT-mTOR and Ras-RafMEK-ERK pathways have been identified as the most

Correspondence to: Dr Jussi Koivunen, Department of Medical Oncology and Radiotherapy and Medical Research Center Oulu, Oulu University Hospital and University of Oulu, Oulu, PB22 90029 OYS, Finland

E-mail: jussi.koivunen@ppshp.fi

Abbreviations: cPARP, cleaved PARP; MEKi, MEK inhibition; MTSassay,3-(4,5-dimethylthiazol-2-yl)-5-(3-carboxymethoxyphenyl)2-(4-sulfophenyl)-2H-tetrazolium)-based cytotoxicity assay; NSCLC, non-small cell lung cancer; PI3Ki, PI3K inhibition

Key words: targeted cancer therapy, PI3K inhibition, MEK inhibition, drug resistance, solid malignancies important oncogenic pathways. Considering the central role of the pathways in transmitting upstream oncogenic signals, their inhibition could be an effective therapy as regards various cancer genotypes (1).

The clinical efficiency of single pathway inhibitors of PI3K-AKT-mTOR and Ras-Raf-MEK-ERK has generally been disappointing, with some exceptions such as their use in cases of $B$-Raf mutant melanoma. Cancers can be de novodependent concurrently on these parallel pathways and cross-signaling of the pathways is also evident $(2,3)$. Many in vivo and in vitro studies have shown that the PI3K-AKTmTOR and Ras-Raf-MEK-ERK pathways regulate each other's activity through feedback mechanisms and have shared downstream targets $(4,5)$. Interaction of the PI3K-AKT-mTOR and Ras-Raf-MEK-ERK signaling pathways is thought to explain the inefficiency of single agent treatments and provide a rationale for the concurrent targeting of both pathways.

Preclinical studies have shown that dual targeting with PI3K and MEK inhibitors has antitumor activity in various cancer models and genotypes (6-8). Many preclinical studies have concerned predictive factors as regards dual PI3K and MEK inhibitor therapy, but so far no clear factors have been identified $(8,9)$. Numerous early-phase clinical studies concerning dual PI3K and MEK targeting are ongoing and some results have recently been presented. Generally, combined PI3K and MEK inhibitor therapy seems to be feasible but, unfortunately, the rate of response is low (10-12). In preclinical models, the vast majority of cancer cell lines do not show apoptosis in response to dual PI3K and MEK targeting, which could be a major factor behind the limited clinical activity of the approach $(8,9)$. Some recent investigations have revealed that drugs affecting the apoptotic pathways, such as $\mathrm{BH} 3$ mimetics, could dramatically increase the rate of apoptosis and the efficiency of MEK and/ or PI3K/mTOR inhibitors (13,14). Bcl-2 family members have been suggested to be important determinants of cell fate in targeted cancer therapies. Induction of pro-apoptotic proteins such as BIM is often linked to apoptosis, while anti-apoptotic proteins such as Mcl-1 and Bcl-xl promote cell survival. The general balance between anti- and pro-apoptotic mediators is crucial for the determination of cell survival or apoptosis (15).

The current study builds upon our earlier work (9) where we identified three cell lines from solid tumors, which showed sensitivity to dual PI3K and MEK blockage but with limited apoptotic response. In this study, we investigated in vitro with 
these cell lines if an additional agent could increase efficiency and apoptotic response to dual PI3K and MEK blockage. We identified some pharmacological agents that could enhance the cytotoxicity of dual PI3K and MEK blockage, and more importantly, induce marked apoptosis. Furthermore, Bcl-xl (Bcl2L1) and Mcl-1 were identified as being important determinants of cell fate.

\section{Materials and methods}

Cell lines. The cell lines used in the current study included the triple-negative NSCLC (non-small cell lung cancer) cell line H1437, the basal-like breast cancer line MDA-MB231 and the K-Ras mutant colorectal cell line HCT116. The NSCLC cell line H1437 was a kind gift from Dr Pasi Jänne (DanaFarber Cancer Institute, Boston, MA, USA) and the breast and colorectal lines (MDA-MB231 and HCT116) were from Dr Peppi Koivunen (Oulu University, Oulu, Finland). The cell lines were cultured in RPMI-1640 supplemented with fetal bovine serum (10\%) plus penicillin and streptomycin $(100 \mathrm{IU} / \mathrm{ml})$. All the cell culture reagents were purchased from HyClone (Logan, UT, USA).

Inhibitors. Pharmacological agents used in the study and their final concentrations are listed in Table I. The drugs were dissolved in DMSO to a concentration of $10 \mathrm{mmol} / \mathrm{l}$, except for cisplatin, which was diluted in distilled water. All the agents were stored in aliquots at $-20^{\circ} \mathrm{C}$. Further dilutions were made in the cell culture medium.

MTS cell viability/cytotoxicity analysis. Cells were plated onto 96-well plates with three or six parallel wells for each treatment, the experiments being replicated at least twice. The following day the cells were drug treated for $72 \mathrm{~h}$, after which the plates were developed using an MTS reagent mix ([3-(4, 5-dimethylthiazol-2-yl)-5-(3-carboxymethoxyphenyl)2-(4-sulfophenyl)-2H-tetrazolium, inner salt], Promega, Madison, WI, USA) supplemented with phenazine methosulfate (Sigma-Aldrich, St. Louis, MO, USA) according to the manufacturer's guidelines and the absorbance was read on a plate reader (Athos Labtec Instruments, Salzburg, Austria) at a wavelength of $488 \mathrm{~nm}$. Graphic display of the data was carried out using GraphPad Prism software (GraphPad Software, La Jolla, CA, USA). Absorbance in the non-treated wells was set as the reference value $(100 \%)$.

Western blot analysis. The cells were plated onto 6-well plates and treated with the drugs $24 \mathrm{~h}$ later for the desired time, after which they were lysed in RIPA buffer [1\% Igepal CA-630, $20 \mathrm{mM}$ Tris- $\mathrm{HCl} \mathrm{pH}$ 8.0, $137 \mathrm{mM} \mathrm{NaCl}, 10 \%$ glycerol, $2 \mathrm{mM}$ EDTA, $1 \mathrm{mM}$ sodium orthovanadate, aprotinin $(10 \mu \mathrm{g} / \mathrm{ml})$, leupeptin $(10 \mu \mathrm{g} / \mathrm{ml})$ and pepstatin $(10 \mu \mathrm{g} / \mathrm{ml})]$. Protein concentrations were measured by Bio-Rad Protein Assay (Bio-Rad, Hercules, CA, USA) and the concentrations in individual samples were equalized before adding $3 \mathrm{X}$ Laemmli buffer to a final concentration of $1 \mathrm{X}$. Equal amounts of protein were run on $12 \%$ SDS-PAGE gels, transferred to PVDF membranes, probed with the antibodies and developed using the ECL chemiluminescence system (Millipore, Billerica, MA, USA) for detection on radiographic film, which was scanned to an
Table I. Agents studied in combination with dual PI3K and MEK inhibition.

\begin{tabular}{lll}
\hline Inhibitor & Target/Class & Concentration in $\mu \mathrm{M}$ \\
\hline ABT-263 & BH3 & $0.1,1$ \\
Afatinib & HER2 & 1 \\
AG-1024 & IGF1R1 & 10 \\
5-azacytidine & Methylation & 1 \\
AZD-2281 & PARP & $0.1,1$ \\
Cis-platin & Chemo & 1 \\
Crizotinib & ALK & 1 \\
Dasatinib & Multi-TKI & 1 \\
Entinostat & Chemo & 1 \\
GDC-0449 & Hedgehog & 10 \\
Gö9976 & PKC & 1 \\
IPI-504 & HSP90 & 1 \\
LY-2157229 & TGF- $\beta$ R1 & 1 \\
Metformin & Diabetics & 100 \\
NVP-XAV939 & WNT & 1 \\
Paclitaxel & Chemo & 0.01 \\
Salinomycin & CSC & $0.1,1$ \\
SD208 & TGF- $\beta$ R1 & 1 \\
Sorafenib & Multi-TKI & 1 \\
Sunitinib & Multi-TKI & 1 \\
DBC & $\gamma$-Secretase & 1 \\
\hline & & 1 \\
\hline
\end{tabular}

electronic format. Primary antibodies to the following proteins were used in the current study: cleaved PARP, Mcl-1, BIM, Bcl-xl and $\beta$-actin. All the primary antibodies were used at a 1:1000 dilution in 5\% BSA. Anti-mouse ( $\beta$-actin) or anti-rabbit (others) IgG HRP-linked antibody was used as the secondary antibody. All antibodies were from Cell Signaling Technology (Danvers, MA, USA).

Immunoprecipitation. For immunoprecipitation, cells were plated on 6-well or $10-\mathrm{cm}$ diameter plates and drug treated the following day for the desired time, after which the cells were lysed in RIPA buffer and protein concentrations were determined by Bio-Rad Protein Assay. Protein $(200 \mu \mathrm{g})$ at a concentration of $1 \mathrm{mg} / \mathrm{ml}$ was incubated overnight at $4^{\circ} \mathrm{C}$ with primary antibody at a 1:100 concentration. The next day protein A/G agarose beads (Santa Cruz Biotechnology, Dallas, TX, USA) were added to each sample and incubated for 1-3 $\mathrm{h}$ at $4^{\circ} \mathrm{C}$, after which the samples were pelleted by centrifugation. The pellets were washed with cell lysis buffer, resuspended in Laemmli buffer and analyzed with western blotting.

siRNA knockdown. For the knockdown studies, BCL-XL (BCL2L1) and MCL-1 (MCL1) specific siRNAs (Dharmacon smart pools) were used at $25 \mathrm{nmol} / \mathrm{l}$ and transfected with DharmaFECT transfection reagent (both from Dharmacon, Lafayette, CO, USA). The experiments were performed according to the manufacturer's protocol. In short, the cells were plated on 96- (for MTS) or 6-well (for western blotting) plates in non-antibiotic media. After $24 \mathrm{~h}$ the medium was changed 

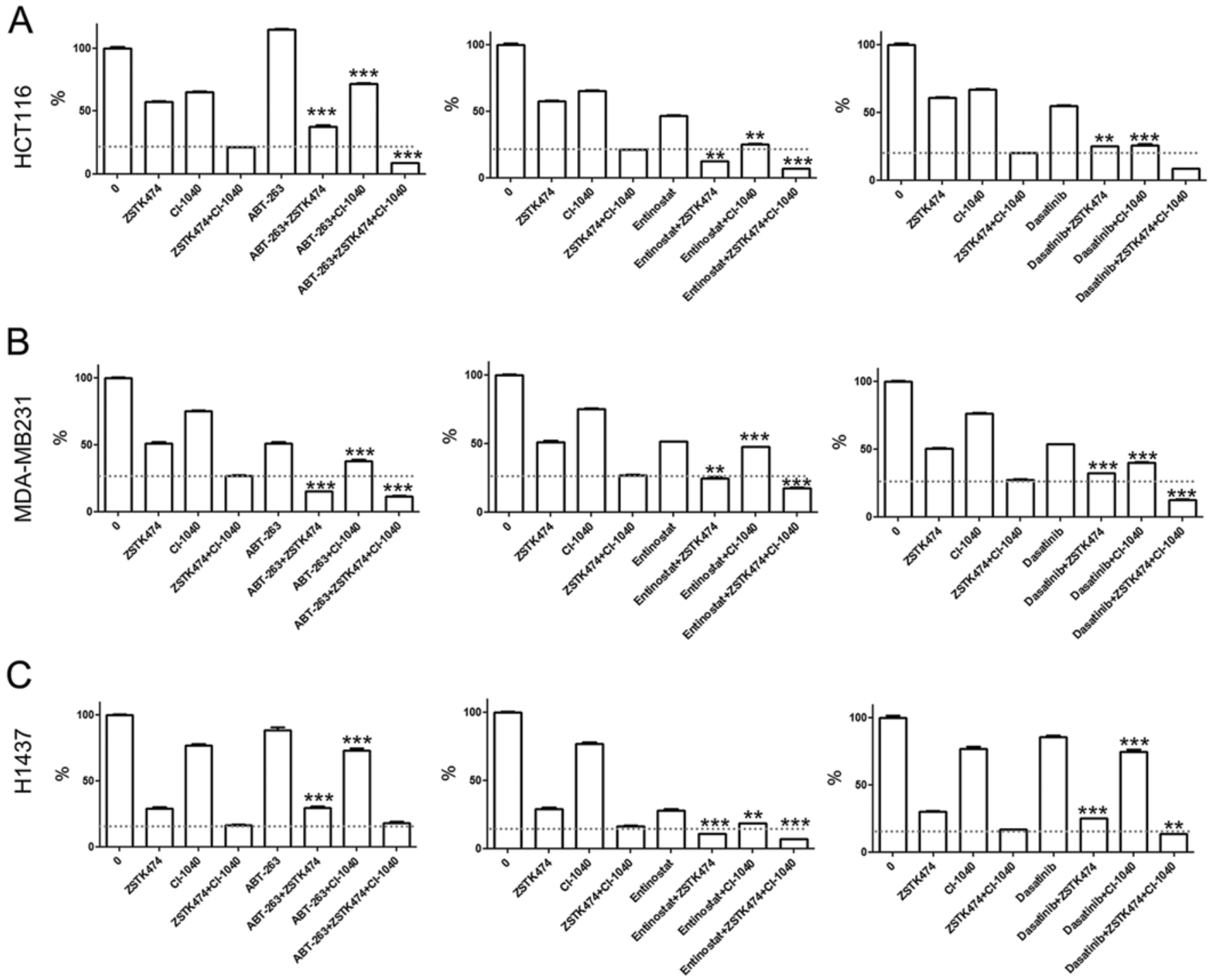

Figure 1. ABT-263, entinostat and dasatinib increase the cytotoxicity of dual PI3K and MEK inhibition. Cells were exposed to the inhibitors for $72 \mathrm{~h}$ and analyzed by using the MTS cytotoxicity assay. Inhibitors were used at the following concentrations: ZSTK474 $(3.3 \mu \mathrm{M})$, CI-1040 $(1 \mu \mathrm{M})$, ABT-263 $(1 \mu \mathrm{M})$, entinostat $(1 \mu \mathrm{M})$ and dasatinib $(0.1 \mu \mathrm{M})$. (A) HCT116 colorectal cancer line. (B) MDA-MB231 breast cancer line. (C) H1437 non-small cell lung cancer line. The Y-axis indicates the cell viability as percentage relative to untreated cells. Statistically significant differences are indicated by asterisks, ${ }^{* *} \mathrm{p}<0.01$; ${ }_{* * * *} \mathrm{p}<0.001$. Error bars indicate standard deviation.

to medium containing siRNA+lipid, or scramble siRNA+lipid (controls), prediluted in Opti-MEM (Gibco/Invitrogen, Carlsbad, CA, USA). The following day, the medium was changed to normal medium. Incubation was continued for the desired time (total of 24-96 h) before assays.

Statistical analysis. Student's two-tailed t-test was used for the statistical analysis. For the analysis, percentage change compared to PI3Ki+MEKi treated (Fig. 1) or scramble siRNA treated cells (knockdown studies) was used. p-values $<0.001$ and $<0.01$ are indicated.

\section{Results}

ABT-263, entinostat and dasatinib increase the cytotoxicity of dual PI3K and MEK inhibition. The effect of combining dual PI3K and MEK inhibition with other pharmacological agents was studied in HCT116 (colon cancer), MDA-MB231 (breast cancer) and H1437 (NSCLC) cell lines, which are known to be sensitive to dual PI3K and MEK inhibition but to show limited apoptotic responses to the treatments (9). The cells were exposed to dual PI3K and MEK inhibition (ZSTK474 and CI-1040) in combination with a panel of other small-molecule inhibitors and cell viability was analyzed by using 72-h MTS cytotoxicity assays (Table I). The concentrations chosen for each drug were based on our preliminary experiments, where we used MTS assay to determine the lowest concentration causing the maximal cytotoxic effect (not shown). Of the 21 tested drugs, the BH3 mimetic ABT-263, the HDAC inhibitor entinostat and the multikinase inhibitor dasatinib were observed to decrease cell viability compared with dual blockage alone, excluding the H1437 line and ABT-263 treatment combination. Of these three drugs, entinostat and dasatinib had single agent cytotoxic activity, while ABT-263 was active only in the MDA-MB231 line. ABT-263, entinostat and dasatinib were chosen for further studies.

We next assessed whether concurrent dual blockage is needed for cytotoxicity of the other agent or if single PI3K or 


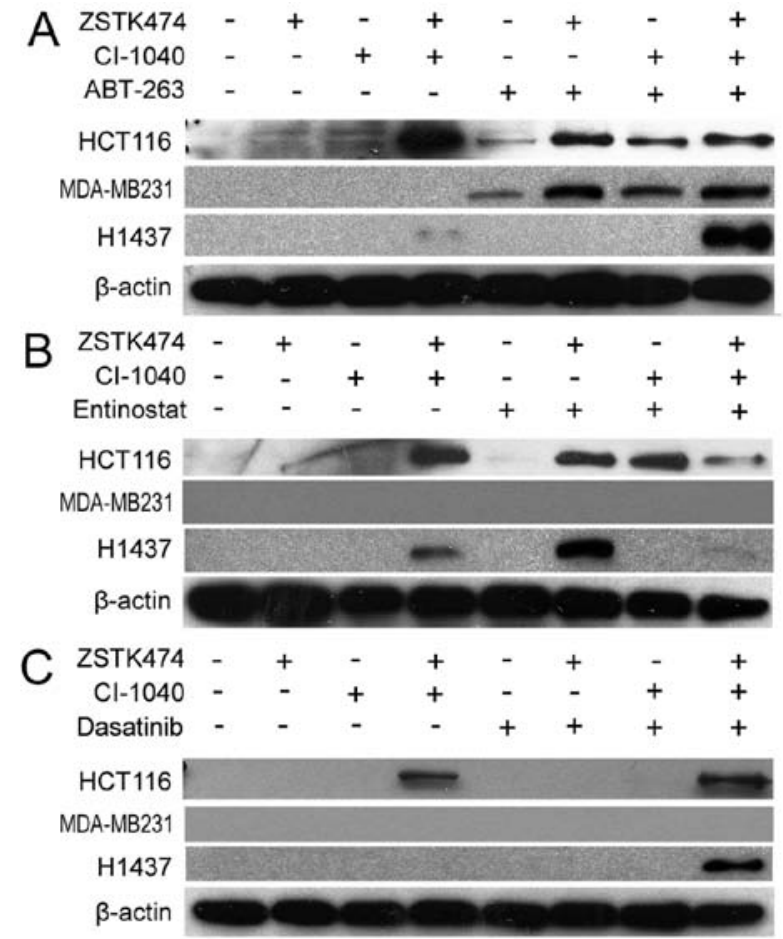

Figure 2. Apoptotic response to dual PI3K and MEK inhibition in combination with ABT-263, entinostat or dasatinib. Western blot analysis of cleaved PARP (cPARP) in response to treatment with ZSTK474 (3.3 $\mu \mathrm{M})$, CI-1040 $(1 \mu \mathrm{M})$, ABT-263 $(1 \mu \mathrm{M})$, entinostat $(1 \mu \mathrm{M})$ or dasatinib $(0.1 \mu \mathrm{M})$ or their combinations for $6 \mathrm{~h}$ (MDA-MB231 cells), $48 \mathrm{~h}$ (HCT116 cells) or $72 \mathrm{~h}$ (H1437 cells). (A) ABT-263-containing treatments. (B) Entinostat-containing treatments. (C) Dasatinib-containing treatments.

MEK inhibition is sufficient, using MTS cytotoxicity assays. In the HCT116 line, ABT-263 was seen to decrease viability in combination with PI3K inhibition (PI3Ki) but not with MEK inhibition (MEKi). Conversely, when HCT116 cells were treated with entinostat or dasatinib, concurrent administration of either PI3Ki or MEKi decreased cell viability (Fig. 1A). In the MDA-MB231 line, a marked increase in cytotoxicity was seen with concurrent administration of ABT-263 or dasatinib with ZSTK474 or CI-1040. Synergistic cytotoxicity was also seen with entinostat co-administration with PI3Ki but not with MEKi (Fig. 1B). In the H1437 line, entinostat was the only agent noted to increase cytotoxicity in combination with either PI3Ki or MEKi, while no marked change was seen with ABT-263 or dasatinib combinations (Fig. 1C).

ABT-263, entinostat and dasatinib increase apoptotic responses to dual PI3K and MEK inhibition. Next we investigated whether the cytotoxic responses seen in MTS assays are accompanied by apoptosis. Western blot analysis of PARP cleavage was used to assess apoptosis after 6-72 h of drug treatments. Based on our preliminary experiments, the rate of the apoptotic response varied dramatically in the tested lines. MDA-MB231 responded with apoptosis within a few hours of the initiation of treatment, while the two other lines responded in days. Therefore, a specific time point for each line was selected. In the HCT116 and MDA-M231 cell lines, marked PARP cleavage was seen when ABT-263 was combined with PI3Ki, MEKi, or their combination. In the H1437 cell line, PARP cleavage was seen only when ABT-263 was combined with dual PI3K and MEK blockage (Fig. 2A). The HCT116 line responded to entinostat treatment analogously to ABT-263, with detectable cleaved PARP when entinostat was co-administered with PI3Ki, MEKi, or their combination. In the H1437 line, cleaved PARP was detected with the entinostat and PI3Ki combination, but not with the other treatments tested. No apoptosis was seen in the MDA-MB231 line with entinostat combinations (Fig. 2B). Dasatinib was able to induce marked PARP cleavage in the H1437 cell line only when combined with dual PI3K and MEK blockage, and not in the other combinations tested (Fig. 2C).

Downregulation of $\mathrm{Bcl}-\mathrm{xl}$ and $\mathrm{Mcl}-1$ correlates with apoptosis in cells treated with PI3K and MEK dual blockage. We further analyzed the effects of the inhibitor treatments on the pro-apoptotic protein BIM and the anti-apoptotic proteins Mcl-1 and Bcl-xl. HCT116 and H1437 lines were selected for the analysis since these cell lines respond to treatment within days and are more reliably assessable as regards expression. Conversely, the MDA-MB231 line undergoes very rapid apoptosis and on the basis of the results of our preliminary experiments, protein levels remain unaltered in this line and therefore we excluded it from these experiments. In HCT116 cells, BIM upregulation was detected in response to all treatments except for co-targeting with ABT-263 or entinostat plus dual PI3K and MEK blockage. Furthermore, there was a tendency for BIM upregulation to occur with single agent MEK inhibition.

In H1437 cells, BIM upregulation correlated with MEK inhibition, but, surprisingly, this was absent when PI3Ki was co-administered. In the HCT116 line, Mcl-1 upregulation was noted in connection with ABT-263 and its combinations and downregulation was seen when dual PI3K and MEK blockage was combined with ABT-263, entinostat, or dasatinib. In the H1437 line, Mcl-1 downregulation correlated with PI3K inhibition. In HCT116 cells, Bcl-xl downregulation was noted in entinostat combinations, and in ABT-263 and dual PI3K and MEK blockage treatments. In the H1437 line, Bcl-xl downregulation was seen with ABT-263 combinations, and with entinostat+PI3Ki and dasatinib+PI3Ki+MEKi. In general, Bcl-xl downregulation does not indicate apoptosis but is required in most cases. As an exception, we did not see Bcl-xl downregulation when the HCT116 line was treated with apoptosis-inducing ABT-263 combinations, but since ABT-263 is an indirect inhibitor of the protein, this is not surprising (Fig. 3A-C).

BIM hetorodimerization with $\mathrm{Bcl}-\mathrm{xl}$ and $\mathrm{Mcl}-\mathrm{I}$ in response to dual PI3K and MEK blockage. Next, we assessed whether there is a difference in Bcl-2 family member dimers in cells treated with PI3K and MEK inhibitor combination. After drug treatments, the cell lysate were immunoprecipitated with BIM antibody and detected with Mcl-1 or Bcl-xl antibodies. In HCT116, dual PI3K and MEK targeting increased incorporation of Bcl-xl to BIM while Mcl-1 levels remained low. In H1437, we saw an increase in Bcl-xl and Mcl-1 incorporation to BIM, but some increase in the Mcl-1 attachment. In MDA-MB231, we could not detect any increase in Bcl-xl or Mcl-1 attachment to BIM in response to dual targeting. This could be related to the short period of drug treatment and quick 

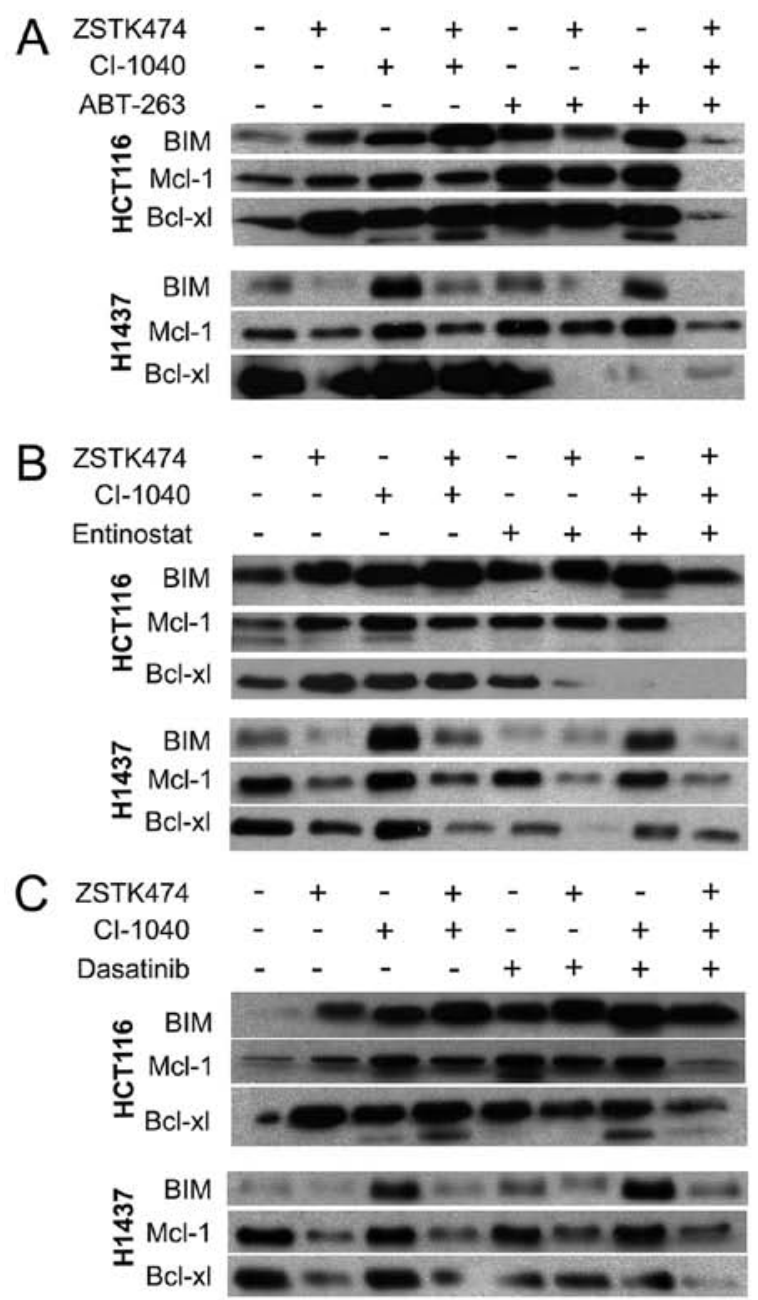

$\mathrm{ZSTK} 474$
$\mathrm{Cl}-1040 \quad+\quad+$
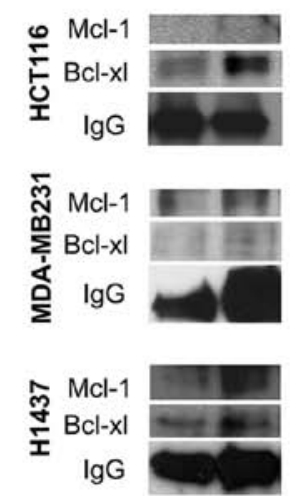

Figure 3. Bcl-xl, BIM and Mcl-1 in response to dual PI3K and MEK inhibition and in combination with ABT-263, entinostat or dasatinib. (A-C) Western blot analysis of BIM, Bcl-xl and Mcl-1 in response to treatments with ZSTK474 (3.3 $\mu \mathrm{M})$, CI-1040 $(1 \mu \mathrm{M})$, ABT-263 $(1 \mu \mathrm{M})$, entinostat $(1 \mu \mathrm{M})$ or dasatinib $(0.1 \mu \mathrm{M})$ and their combinations for $48 \mathrm{~h}$ (HCT116 cells) or $72 \mathrm{~h}$ (H1437 cells). (D) Immunoprecipitation with BIM antibody followed by detection using Bcl-xl or Mcl-1 antibodies in untreated cells or cells treated with dual ZSTK474 and CI-1040 for 2 (MDA-MB231 cells), 24 (HCT116 cells), or 48 h (H1437).

apoptotic response seen with pharmacological Bcl-2/Bcl-xl blockage in this specific cell line (Fig. 3D).

Knockdown of Bcl-xl expression increases the cytotoxicity of dual PI3K and MEK blockage. HCT116, MDA-MB231 and H1437 cells were subjected to $B C L-X L$-specific siRNA knockdown. The cells were first analyzed by western blotting for Bcl-xl expression. In MDA-MB231 cells we saw some downregulation of Bcl-xl after 24- and 48-h treatment, while almost complete absence of the protein was noted following 72- and 96-h treatments. In the HCT116 and H1437 lines, 72- or 96-h treatment with $B C L-X L$-specific siRNA induced downregulation of $\mathrm{Bcl}-\mathrm{xl}$ expression, but this, however, was lower in the H1437 line when compared with the MDA-MB231 line (Fig. 4A).

Based on the MTS cytotoxicity assay, siRNA knockdown of $B C L-X L$ was not cytotoxic by itself in any of the tested lines. To evaluated whether $B C L-X L$ knockdown would sensitize the cells to dual PI3K and MEK blockage, the cells were pretreated with or without $B C L-X L$ siRNA for 24-48 h, after which they were exposed to PI3Ki, MEKi, or their combination for an additional $72 \mathrm{~h}$ and analyzed by using MTS cytotoxicity assays. Increased cytoxicity was seen in the MDA-MB231 line with PI3Ki and/or MEKi treatment after $B C L-X L$ knockdown. This was not observed in the other two cell lines tested except for single agent MEKi in the HCT116 cell line (Fig. 4B). We then investigated whether $B C L-X L$ knockdown would increase apoptosis in response to PI3Ki and/or MEKi. In HCT116 cells, PARP cleavage was evident in PI3Ki and more notably, in MEKi treated cells after $B C L-X L$ knockdown, but not in control cells. Furthermore, a marked increase in the cleaved PARP signal was seen after dual PI3K and MEK targeting when BCL-XL was suppressed. In the MDA-MB231 line, PARP cleavage was evident in cells treated with PI3Ki and/or MEKi combination in the cells with $B C L-X L$ knockdown, but not in controls (Fig. 4C). In H1437 cells, we did not see a marked change in the PARP cleavage profile in cells with $B C L-X L$ knockdown as expected (not shown).

Knockdown of MCl-1 expression increases the cytotoxicity of dual PI3K and MEK blockage. Since H1437 line showed increased attachment of Mcl-1 to BIM in response to dual PI3K and MEK targeting and was less sensitive to pharmacological or siRNA blockage of $B C L-X L$, we assessed whether knockdown of $\mathrm{MCl}-\mathrm{l}$ on would increase the cytotoxic and apoptotic response in this cell line. HCT116, MDA-MB231, and $\mathrm{H} 1437$ lines were exposed to siRNA-mediated knockdown of $\mathrm{MCl}-1$. Marked decrease of Mcl-1 expression was noted in cells treated with $\mathrm{MCl}$-1-specific siRNA but not with scramble siRNA (Fig. 5A). Based on MTS cytotoxicity assay, siRNA knockdown of $\mathrm{MCl}-\mathrm{l}$ was not cytotoxic by itself in any of the tested cell lines. In the HCT116 line, knockdown of $\mathrm{MCl}-1$ had no synergistic cytotoxic effect with the drugs tested. In the MDA-MB231 cell line, $\mathrm{MCl}-1$ knockdown was able to provoke increased cytotoxicity with all the tested agents. The most dramatic difference was seen in MDA-MB231 cells treated with ABT-263 as a single agent where control cells showed some cytotoxicity, which was highly promoted by MCl-1 knockdown. In the H1437 cell line, siRNA knockdown of $\mathrm{MCl}-1$ was able to induce significant cytotoxicity in treatment regimens containing $\mathrm{PI} 3 \mathrm{Ki}$ and with MEKi+ABT-263 combination (Fig. 5B).

Next, we analyzed whether $M C l-1$ knockdown would lead to apoptotic response in H1437 and MDA-MB231 lines. In 

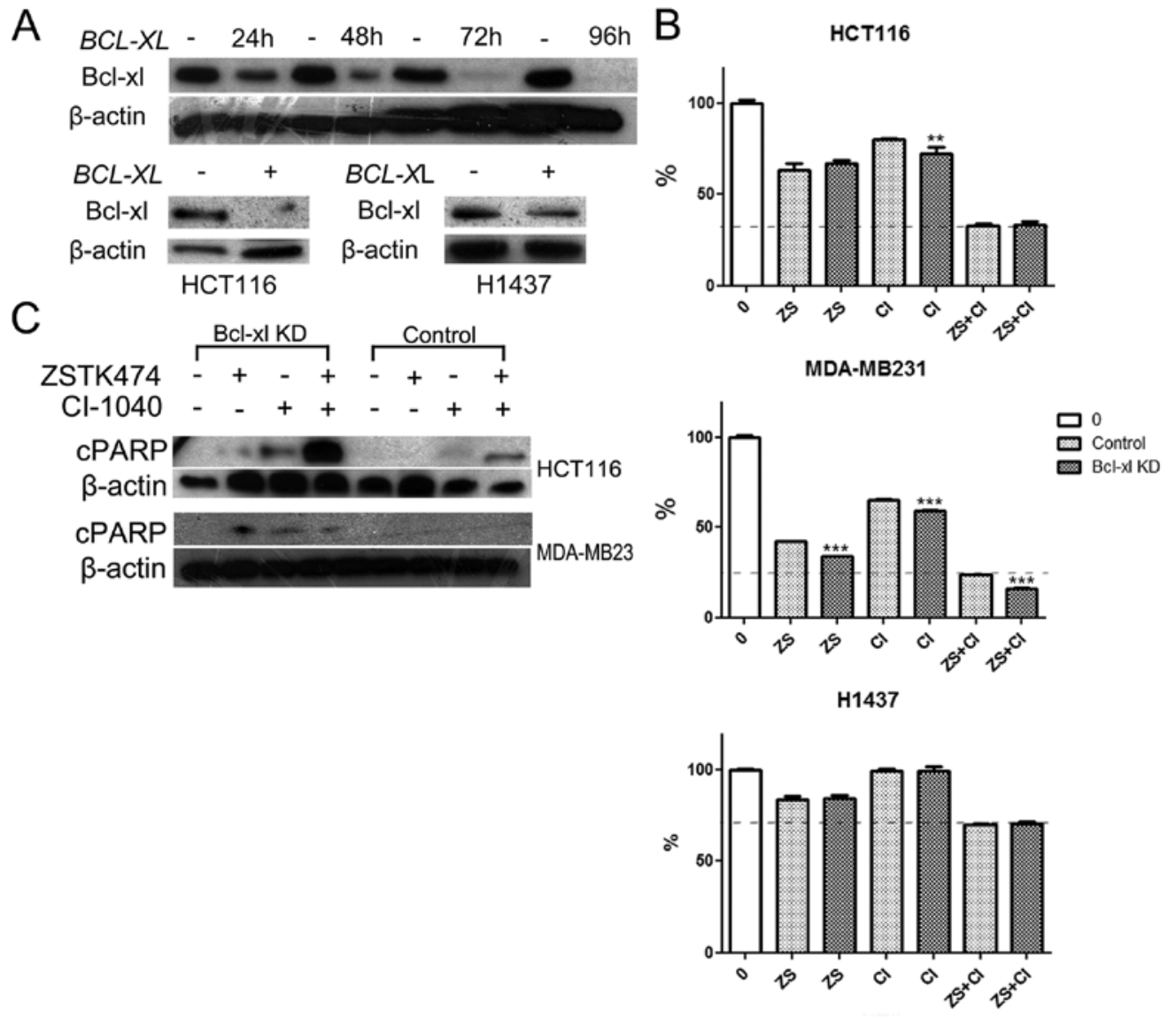

Figure 4. $B C L-X L$ knockdown increases the cytotoxicity of dual PI3K and MEK blockage. Bcl-xl protein expression was downregulated using siRNA-mediated knockdown of the gene. (A) Expression of Bcl-xl after treatment of cells with (BCL-XL) or without (control) $B C L-X L$ siRNA in MDA-MB231, HCT116 and H1437 lines. (B) MTS cytotoxicity assay of cells pretreated with (Bcl-xl KD) or without (control) $B C L-X L$ siRNA for $24 \mathrm{~h}$, after a further 72-h treatment with ZSTK474, CI-1040, or their combination. The Y-axis indicates the cell viability as percentage relative to untreated cells. Statistically significant differences are indicated by asterisks, ${ }^{* *} \mathrm{p}<0.01,{ }^{* * *} \mathrm{p}<0.001$. Error bars indicate standard deviation. (C) Western blot analysis of cleaved PARP after treatment with ZSTK474, CI-1040, or their combination in cells pretreated with or without $B C L-X L$ siRNA for $24 \mathrm{~h}$.

MDA-MB231 cells apoptosis was evident in the cells treated with ABT-263 containing treatment regimens but expression of cleaved PARP was increased after $\mathrm{MCl}-1$ knockdown. In the H1437 cell line, a trace of PARP cleavage was evident only in cells treated with PI3Ki+MEKi+ABT-263 after scramble siRNA treatment. Conversely, when $\mathrm{MCl}-\mathrm{l}$ was knocked down, marked apoptosis was evident in cells treated with PI3Ki+MEKi, PI3Ki+ABT-263, and PI3Ki+MEKi+ABT-263 (Fig. 5C).

\section{Discussion}

The PI3K-AKT-mTOR and Ras-Raf-MEK-ERK pathways are central transmitters of oncogenic signals in solid malignancies. Considering the central role of the pathways, their inhibition could be an effective therapy in various cancer genotypes. Even though PI3K-AKT-mTOR and Ras-Raf-MEK-ERK are the most commonly altered signaling pathways in solid malignancies, the clinical efficiency of single pathway inhibitors has generally been disappointing and combinatorial approaches have been applied. Preclinical models have shown that dual targeting with PI3K and MEK inhibitors has antitumor activity in various cancer models $(3,8,16,17)$. Numerous early-phase clinical studies concerning dual PI3K and MEK targeting are ongoing and some results have already been presented.
Generally, combined PI3K and MEK inhibitor therapy seems to be feasible, but, unfortunately, the rate of response seems to be low (10-12). In preclinical models, the vast majority of cancer cell lines do not show apoptosis in response to dual PI3K and MEK targeting, which could be the major factor behind the limited clinical activity of the approach $(8,9)$.

In the current work, we employed cell lines identified in our earlier study (9) to evaluate whether the apoptotic response to dual PI3K and MEK inhibition could be augmented by pharmacological means in vitro. In the screen, we found that the Bcl-2/Bcl-xl inhibitor ABT-263, the HDAC inhibitor entinostat and the multikinase inhibitor dasatinib increased the cytotoxic response and apoptosis in combination with PI3K and MEK dual blockage. Analogously to our results, some recent publications have described how Bcl-2/Bcl-xl targeting can enhance the cytotoxicity of MEK and mTOR inhibitors, and dual PI3K and MEK blockage $(13,14)$. Furthermore, HDAC inhibition has earlier been shown to increase the efficiency of both MEK and PI3K-AKT-mTOR targeting agents $(16,18)$. Moreover, earlier research has proposed HDAC inhibitors to act through Mcl-1 (19). To our knowledge, however, previous work provided only limited evidence on the use of dasatinib in combination with PI3K and MEK inhibitors.

It is challenging to include three investigational agents concurrently in a clinical trial, and therefore we were also inter- 

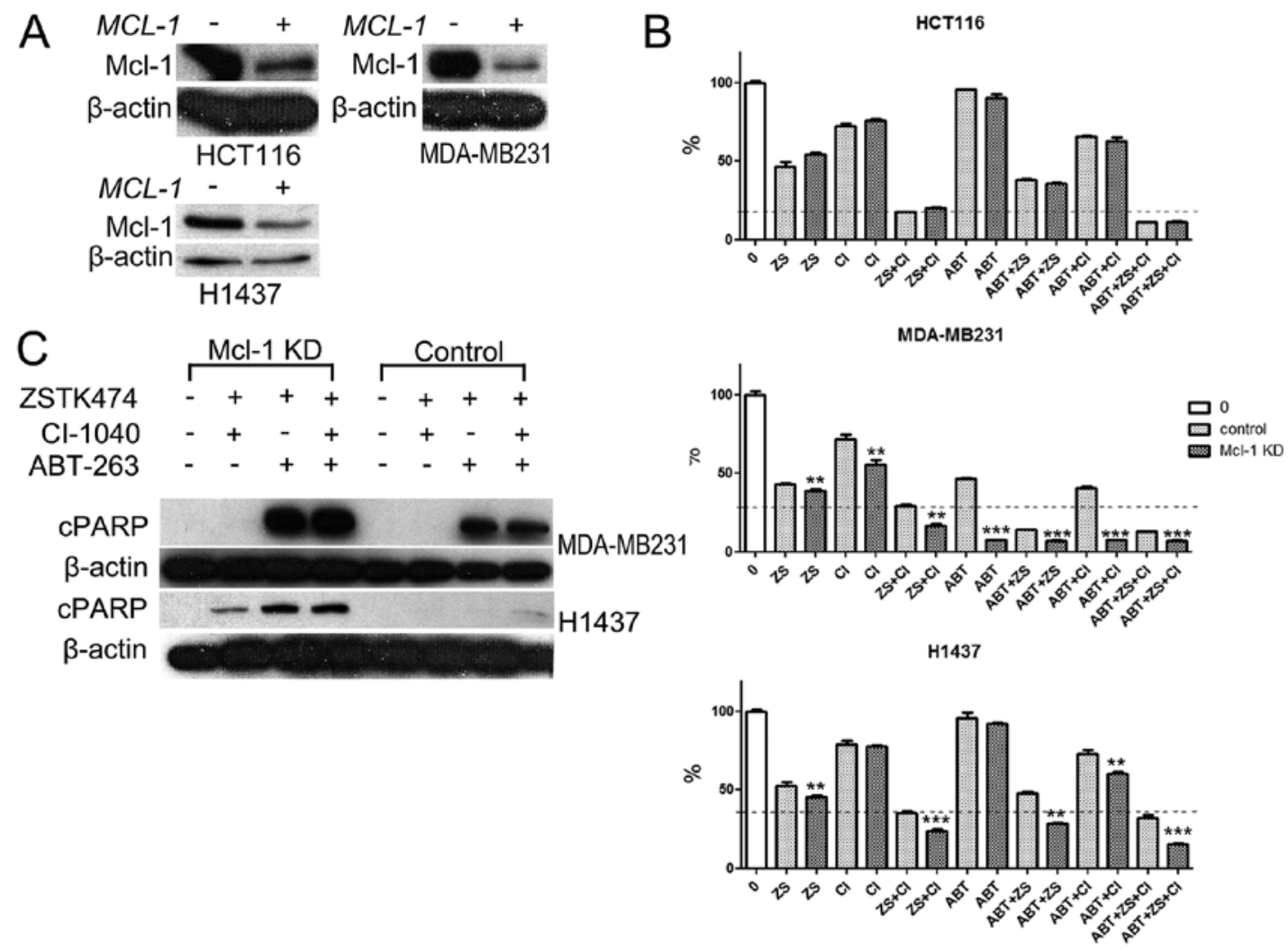

Figure 5. MCL-1 knockdown increases the cytotoxicity of dual PI3K and MEK blockage. Mcl-1 protein expression was downregulated with siRNA-mediated gene knockdown. (A) Mcl-1 expression after treatment with MCl-1 specific or scramble siRNA in HCT116, MDA-MB231 and H1437 lines. (B) MTS cytotoxicity assay of cells pretreated with $\mathrm{MCl}-1$ or scramble siRNA for $24 \mathrm{~h}$, after a further 72-h treatment with ZSTK474, CI-1040, ABT-263 or their combinations. The Y-axis indicates the cell viability as percentage relative to untreated cells. Statistically significant differences are indicated by asterisks, ${ }^{* *} \mathrm{p}<0.01,{ }^{* * * *} \mathrm{p}<0.001$. Error bars indicate standard deviation. (C) Western blot analysis of cleaved PARP after treatment with ZSTK474, CI-1040, ABT-263 or their combinations in cells pretreated with $\mathrm{MCl}-1$ or scramble siRNA for $24-48 \mathrm{~h}$.

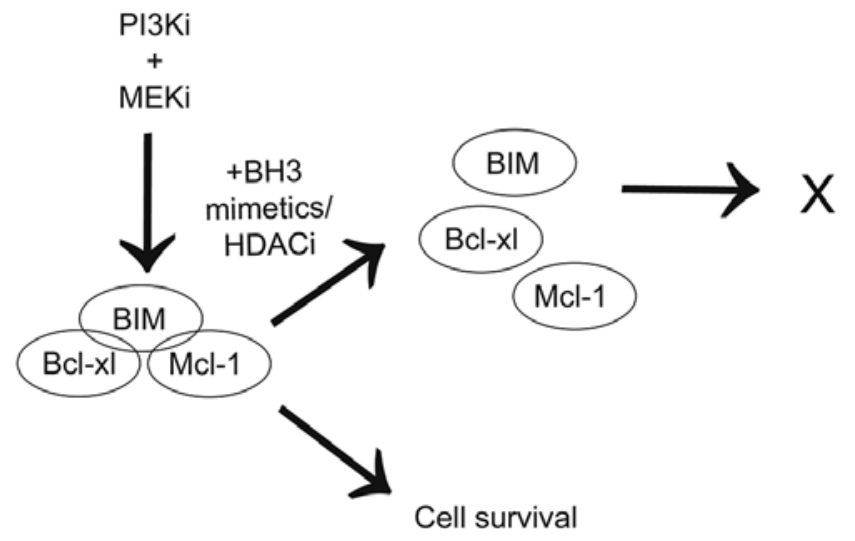

Figure 6. Schematic presentation of anti-apoptotic mechanisms and its pharmacological inhibition in cancers treated with PI3K and MEK inhibitor combination.

ested to see if either PI3K or MEK inhibitor efficiency could be enhanced by combining ABT-263, entinostat or dasatinib to them. All three of these agents were found to increase the cytotoxicity of either the PI3K inhibitor or the MEK inhibitor, excluding ABT-263 in the H1437 line. Apoptotic response was also seen with dual ABT-263 and PI3K or MEK therapy in the HCT116 and MDA-MB231 lines, with entinostat and PI3K or MEK therapy in the HCT116 line, and with entinostat and PI3K therapy in H1437 cells. Based on the results of our preclinical models, it would be appealing to test $\mathrm{Bcl}-\mathrm{xl} / \mathrm{Bcl}-2$ or HDAC inhibitors in combination with PI3K or MEK inhibitors in animal models and clinical trials. Some of these, such as Bcl-2/Bcl-xl and MEK (NCT02079740), and HDAC and mTOR (NCT01087554, NCT01174199) inhibitor combinations, are currently tested in ongoing early phase clinical trials.

Previous studies have identified the apoptotic proteins BIM, Puma, Mcl-1, Bcl-2 and Bcl-xl as major determinants of cell fate in response to PI3K-AKT-mTOR and/or MEK inhibitors. Bcl-xl and Mcl-1 have been suggested to be the most important anti-apoptotic mediators in solid malignancies $(13,14)$. Our results provide similar proof, since drug treatments enabling downregulation of Bcl-xl expression, blockage of its function by the $\mathrm{BH} 3$ mimetic or siRNA gene knockdown resulted in apoptosis in most cases. However, downregulation or blockage of Bcl-xl did not indicate apoptosis but is required in most cases. One of the tested lines (H1437) showed more dependency on both anti-apoptotic proteins Bcl-xl and Mcl-1, since downregulation, blockage, or knockdown of $B C L-X L$ itself was insufficient to cause apoptosis if not accompanied by Mcl-1 downregulation. Furthermore, $\mathrm{MCl}-1$ knockdown in this cell line was able to produce prominent increase in cytotoxicity and apoptosis after treatment with PI3K inhibitor or its combinations. It is likely that many cancer cells are able to circumvent PI3K and/or MEK inhibition-mediated apoptosis. Drug treatments inhibiting Bcl-xl and/or Mcl-1 would, therefore, increase the apoptotic response to PI3K and/or MEK 
inhibitors. In our study, BIM expression showed no correlation to apoptosis.

In the current study, we found that combining Bcl-2/Bcl-xl, HDAC and multikinase inhibitors to PI3K and MEK dual blockage can increase cytotoxicity and apoptosis in vitro. Furthermore, these agents were also able to enhance cytotoxicity and apoptosis of single-agent PI3K and MEK drugs. More importantly, we found Bcl-xl and Mcl-1 to be major determinants of cell fate in connection with PI3K and/or MEK inhibitor treatment (Fig. 6). We conclude that understanding the molecular mechanism of the anti-apoptotic response to dual PI3K and MEK treatment could provide new and smarter pharmacological approaches and lead to more efficient combinations to be tested in clinical trials.

\section{Acknowledgements}

We wish to thank Anne Bisi for her technical assistance. The study was supported by Thelma Mäkikyrö Foundation (J.P.K.), Sigrid Juselius Foundation (J.P.K.), Cancer Society of Northern Finland (E.J.), Ida Montin Foundation (E.J.), and Emil Aaltonen Foundation (E.J.).

\section{References}

1. Hanahan D and Weinberg RA: Hallmarks of cancer: The next generation. Cell 144: 646-674, 2011.

2. Chandarlapaty S, Sawai A, Scaltriti M, Rodrik-Outmezguine V, Grbovic-Huezo O, Serra V, Majumder PK, Baselga J and Rosen N: AKT inhibition relieves feedback suppression of receptor tyrosine kinase expression and activity. Cancer Cell 19: 58-71, 2011.

3. Faber AC, Li D, Song Y, Liang MC, Yeap BY, Bronson RT, Lifshits E, Chen Z, Maira SM, García-Echeverría C, et al: Differential induction of apoptosis in HER2 and EGFR addicted cancers following PI3K inhibition. Proc Natl Acad Sci USA 106: 19503-19508, 2009.

4. Mendoza MC,ErEE and Blenis J: The Ras-ERK and PI3K-mTOR pathways: Cross-talk and compensation. Trends Biochem Sci 36: 320-328, 2011

5. Turke AB, Song Y, Costa C, Cook R, Arteaga CL, Asara JM and Engelman JA: MEK inhibition leads to PI3K/AKT activation by relieving a negative feedback on ERBB receptors. Cancer Res 72: 3228-3237, 2012.

6. Engelman JA, Luo J and Cantley LC: The evolution of phosphatidylinositol 3-kinases as regulators of growth and metabolism. Nat Rev Genet 7: 606-619, 2006.

7. Hoeflich KP, Merchant M, Orr C, Chan J, Den Otter D, Berry L, Kasman I, Koeppen H, Rice K, Yang NY, et al: Intermittent administration of MEK inhibitor GDC-0973 plus PI3K inhibitor GDC-0941 triggers robust apoptosis and tumor growth inhibition. Cancer Res 72: 210-219, 2012.
8. Sos ML, Fischer S, Ullrich R, Peifer M, Heuckmann JM, Koker M, Heynck S, Stückrath I, Weiss J, Fischer F, et al: Identifying genotype-dependent efficacy of single and combined PI3K- and MAPK-pathway inhibition in cancer. Proc Natl Acad Sci USA 106: 18351-18356, 2009.

9. Jokinen E, Laurila N and Koivunen JP: Alternative dosing of dual PI3K and MEK inhibition in cancer therapy. BMC Cancer 12: $612,2012$.

10. Bedard P, Tabernero J, Kurzrock R, et al: A phase lb, openlabel, multicenter, dose-escalation study of the oral pan-PI3K inhibitor BKM120 in combination with the oral MEK1/2 inhibitor GSK1120212 in patients (pts) with selected advanced solid tumors. J Clin Oncol (ASC Annual Meeting abstracts) 30: 3003, 2012.

11. Britten $\mathrm{C}$, Wainberg Z, Tabernero J, et al: A multi-arm phase 1 dose escalation study of safety, pharmacokinetics, and pharmacodynamics of the dual PI3K/mTOR inhibitors PF-04691502 (oral) and PF-05212384 (IV) in combination with the MEK inhibitor PD-0325901 or irinotecan in patients with advanced cancer. Eur J Cancer 48: 109, 2012.

12. LoRusso P, Shapiro G, Pandya SS, et al: A first-in-human phase 1b study to evaluate the MEK inhibitor GDC-0973, combined with the pan-PI3K inhibitor GDC-0941, in patients with advanced solid tumors. J Clin Oncol (ASC Annual Meeting abstracts) 30: 2566,2012

13. Faber AC, Coffee EM, Costa C, Dastur A, Ebi H, Hata AN, Yeo AT, Edelman EJ, Song Y, Tam AT, et al: mTOR inhibition specifically sensitizes colorectal cancers with KRAS or BRAF mutations to BCL-2/BCL-XL inhibition by suppressing MCL-1. Cancer Discov 4: 42-52, 2014.

14. Hata AN, Yeo A, Faber AC, Lifshits E, Chen Z, Cheng KA, Walton Z, Sarosiek KA, Letai A, Heist RS, et al: Failure to induce apoptosis via BCL-2 family proteins underlies lack of efficacy of combined MEK and PI3K inhibitors for KRAS-mutant lung cancers. Cancer Res 74: 3146-3156, 2014.

15. Youle RJ and Strasser A: The BCL-2 protein family: Opposing activities that mediate cell death. Nat Rev Mol Cell Biol 9: 47-59, 2008.

16. Morelli MP, Tentler JJ, Kulikowski GN, Tan AC, BradshawPierce EL, Pitts TM, Brown AM, Nallapareddy S, Arcaroli JJ, Serkova NJ, et al: Preclinical activity of the rational combination of selumetinib (AZD6244) in combination with vorinostat in KRAS-mutant colorectal cancer models. Clin Cancer Res 18: 1051-1062, 2012

17. Wee S, Jagani Z, Xiang KX, Loo A, Dorsch M, Yao YM, Sellers WR, Lengauer C and Stegmeier F: PI3K pathway activation mediates resistance to MEK inhibitors in KRAS mutant cancers. Cancer Res 69: 4286-4293, 2009.

18. Ellis L, Ku SY, Ramakrishnan S, Lasorsa E, Azabdaftari G, Godoy A and Pili R: Combinatorial antitumor effect of HDAC and the PI3K-Akt-mTOR pathway inhibition in a Pten defecient model of prostate cancer. Oncotarget 4: 2225-2236, 2013.

19. He L, Torres-Lockhart K, Forster N,Ramakrishnan S, Greninger P, Garnett MJ, McDermott U, Rothenberg SM, Benes $\mathrm{CH}$ and Ellisen LW: Mcl-1 and FBW7 control a dominant survival pathway underlying HDAC and Bcl-2 inhibitor synergy in squamous cell carcinoma. Cancer Discov 3: 324-337, 2013. 